\author{
A. Torresin ${ }^{1}$, A. Moscato ${ }^{1}$, M. Minella ${ }^{1}$, F. Cardinale ${ }^{2}$, \\ L. Minati ${ }^{3}$, D. Aquino ${ }^{3}$
}

\title{
Fibre tracking: probabilistic approach and preliminary results
}

\footnotetext{
${ }^{1}$ Medical Physics Department, Ospedale Niguarda Ca'Granda, Milano, Italy ${ }^{2}$ Epilepsy Neurosurgery Department, Ospedale Niguarda Ca'Granda, Milano, Italy

${ }^{3}$ Neuroradiological Department, IRCCS C.Besta, Milano, Italy

e-mail: alberto.torresin@unimi.it
}

The aim of this work is to have a preliminary experience with probabilistic tractography. We performed fibres reconstruction for three tracts of interest with data obtained from two MR imaging units equipped with different gradients system. An acquisition protocol optimization has been necessary in order to obtain a good trade-off between image quality and data collection time. Possible solutions to acquisition and processing problems are discussed. Future developments and possible applications in neurosurgery are also suggested.

Key words: probabilistic fibre tracking, DTI, tractography, diffusion imaging.

\section{Introduction}

Diffusion Tensor Magnetic Resonance Imaging provides the unique possibility to probe the cell structure at a microscopic scale. This technique is particularly useful in the neurological field since it allows a $3 \mathrm{D}$ reconstruction of white matter fibres [5]. In this work we made a first attempt to introduce in clinical routine a relative new method to perform the Fibre Tracking based on a probabilistic framework [2]. The novelty introduced by this approach is to consider the measured water molecules principal diffusion direction (PDD) not only equal to the main diffusion tensor eigenvector (as in deterministic approaches), but also associated with an uncertainty. This can be achieved 
generating a probability density function (PDF) for the PDD in each voxel of the imaged volume. The central value of this (PDF) is the principal eigenvector of the Diffusion Tensor. Unfortunately the probabilistic approach is more demanding than the deterministic one, since it requires many diffusion sensitizing directions and a higher signal to noise ratio. This translates in a data collecting time increment. For these reasons we define an optimized acquisition protocol and repeat the entire experiment for two MR imaging units equipped with different gradients system. We want to emphasize the importance of an appropriate parameters tuning (e.g. eddy currents calibration) and the fact that the scanner must have a minimum performance level (e.g. gradients max. strength and slew rate). In front of a constancy in data quality between the two imaging units, we observe a significant reduction in the acquisition time for the scanner equipped with higher performance magnetic field gradients. This time decrease allows to introduce probabilistic tractography in clinical practice. There is another difficulty that has to be fronted in the case of probabilistic fibre tracking: the generation of the (PDF), for each voxel of the volume of interest, requires a lot of time. To overcome this problem it is necessary to use an appropriate hardware which allows high computing performances.

The features offered by the probabilistic approach can lead to possible future developments involving neurosurgery and neurophysiology.

\section{Materials and methods}

\section{Data acquisition}

Diffusion weighted images have been acquired from four healthy volunteers for the first scanner (Philips Achieva 1.5T Nova, max. gradients amplitude $33 \mathrm{mT} / \mathrm{m}$, max. slew rate $160 \mathrm{mT} / \mathrm{m} / \mathrm{s}$, Philips Medical Systems, Best, the Netherlands) and from one volunteer with the second unit (Philips Achieva 1.5T Nova Dual HP, max gradients strength 66 $\mathrm{mT} / \mathrm{m}$, max slew rate $180 \mathrm{mT} / \mathrm{m} / \mathrm{s}$, Philips Medical Systems, Best, the Netherlands). The acquisition protocol has been optimized in order to get a good trade-off between signal-to-noise ratio enhancement, acquisition time and motion artefacts reduction. We used a Spin Echo Single Shot EPI, voxel $2 \mathrm{~mm} \times 2 \mathrm{~mm}$ in-plane with a $112 \times 112$ matrix, $2.6 \mathrm{~mm}$ slice thickness, 50 slices with no gap for a complete brain coverage. Diffusion weighting was applied along 64 directions with a b-value of $1000 \mathrm{~mm}^{2} / \mathrm{s}$. 
A $\mathrm{T}_{2}$-weighted volume with no diffusion weighting was also acquired with the same geometry. In addition, we use the Sense Parallel Imaging scheme (reduction factor: 2) coupled with a six-channels phased-array head coil. This sequence was repeated 8 times with the first scanner (approximately 65 minutes) and 4 times for the second one (32 minutes). Before the processing stage the different acquisitions have been co-registered an averaged in order to obtain a single optimized volume.

\section{Data Processing}

To process images we used FSL [7]. Co-registrations were performed with FLIRT (Fmrib's Linear Registration Tool) [3]; probability density functions were determined for each voxel with BEDPOSTX (Bayesian Estimation of diffusion Parameters using Sampling Techniques) [1]. Fibres reconstruction was performed with PROBTRACK tool [1], working in single seed mask modality, 0.2 curvature threshold, 5000 samples, 2000 maximum number of steps and $0.5 \mathrm{~mm}$ maximum step length. BedpostX required approximately 20 hours with a $2.0 \mathrm{GHz}$ processor. We reconstructed three tracts of interest: Cortico Spinal Tract (CST), Corpus Callosum (CC) and Cingulum (CG). $1 \%$ of the maximum value was used to threshold the obtained tractogram. In this way only the voxels with a very low probability were discarded.

\section{Results and discussion}

In this section we show the obtained results for the three fibre bundles we have chosen. The reconstructed tractograms are depicted along the axial, coronal and sagittal plane and they are superimposed on structural images $\left(\mathrm{T}_{1}\right.$ or $\left.\mathrm{T}_{2}\right)$. In addition the corresponding colour-coded map is also displayed. In Figure 1 we show the results obtained for the CST reconstruction starting from the data acquired with the first scanner (equipped with the $33 \mathrm{mT} / \mathrm{m}$ gradients system). $\mathrm{T}_{1}$ images help to verify the correct anatomical position of the fibre bundle. Reconstructed tracts are depicted in a red-yellow colour-scale: the brighter is a voxel, the higher is the probability that it belongs to a white matter tract connected with the seed mask. Starting from this criterion it is easy to notice that the obtained results show an high probability to be connected with the seed mask. This translates in a high probability of reconstruction 


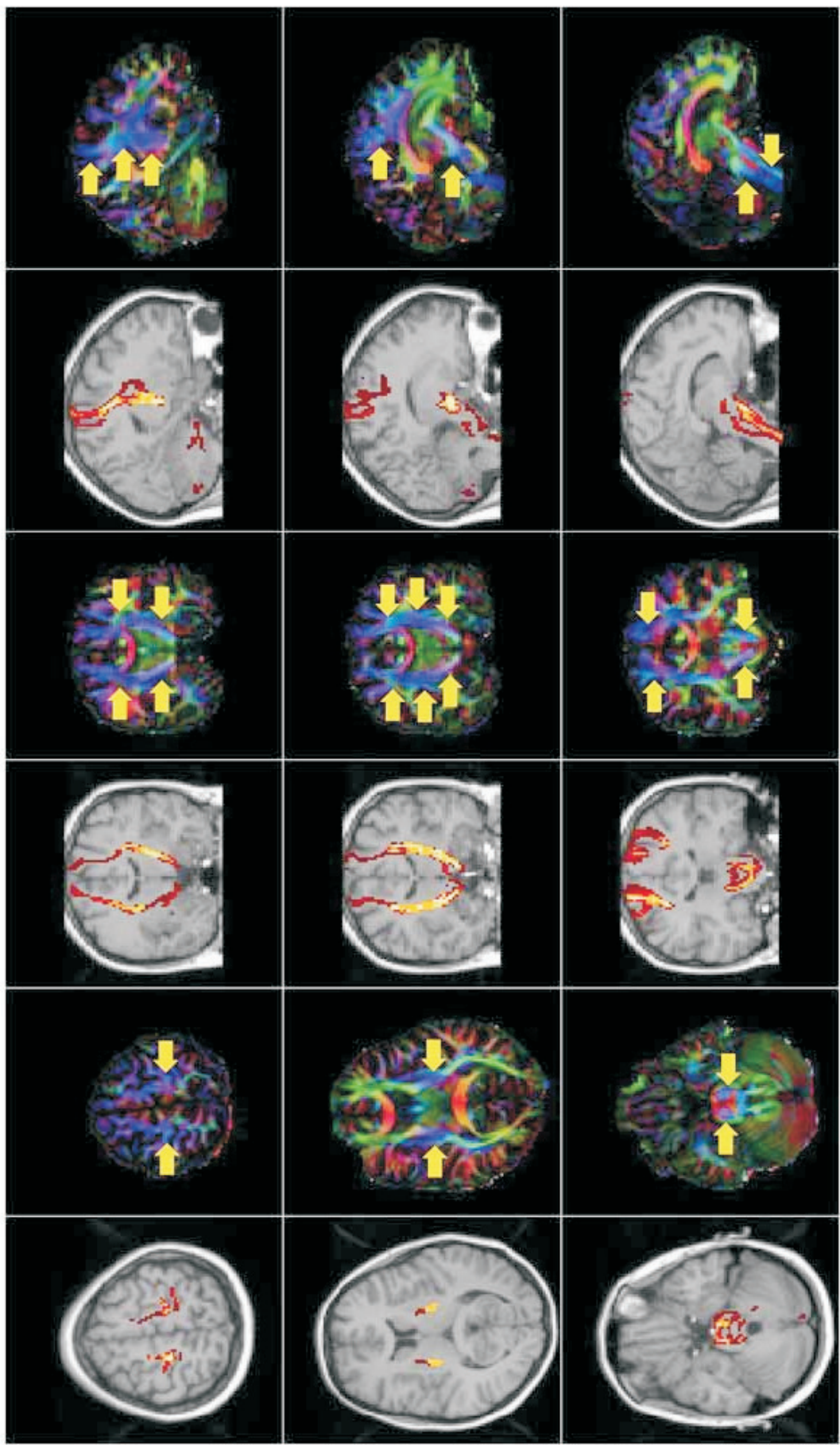

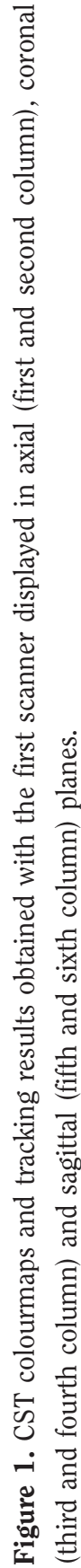




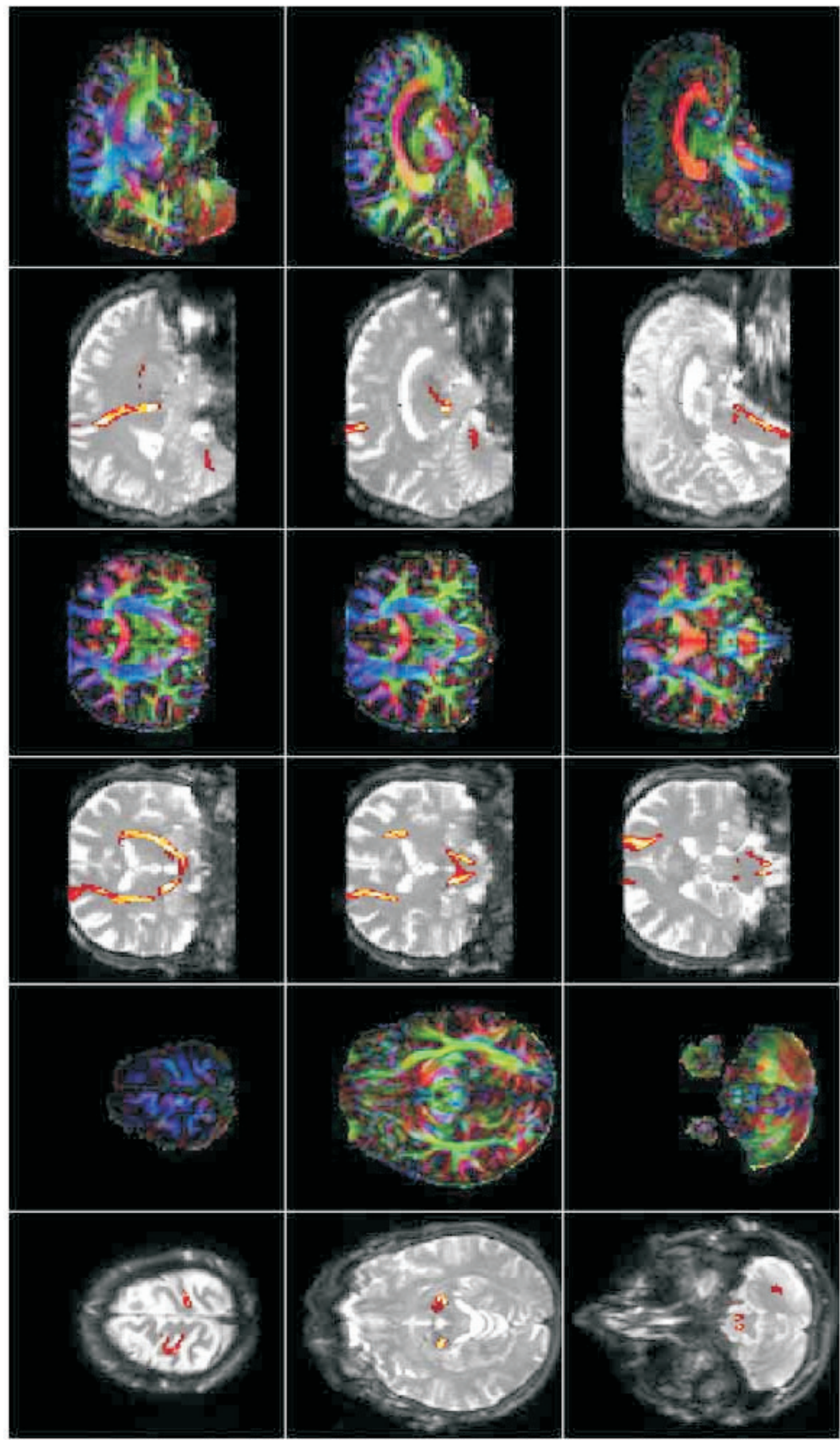

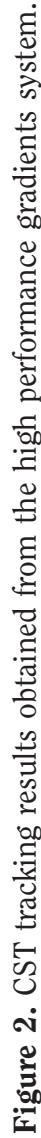




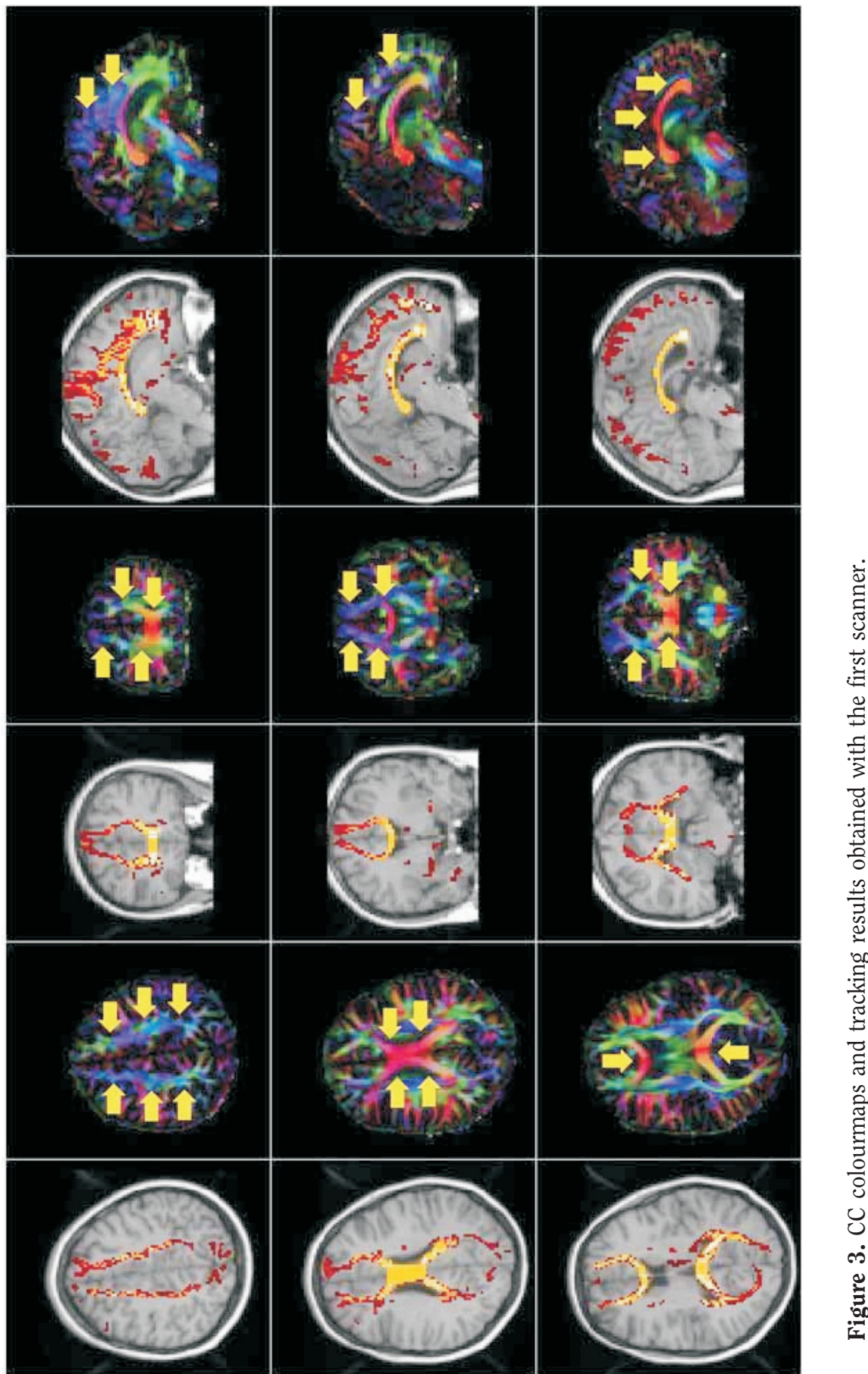




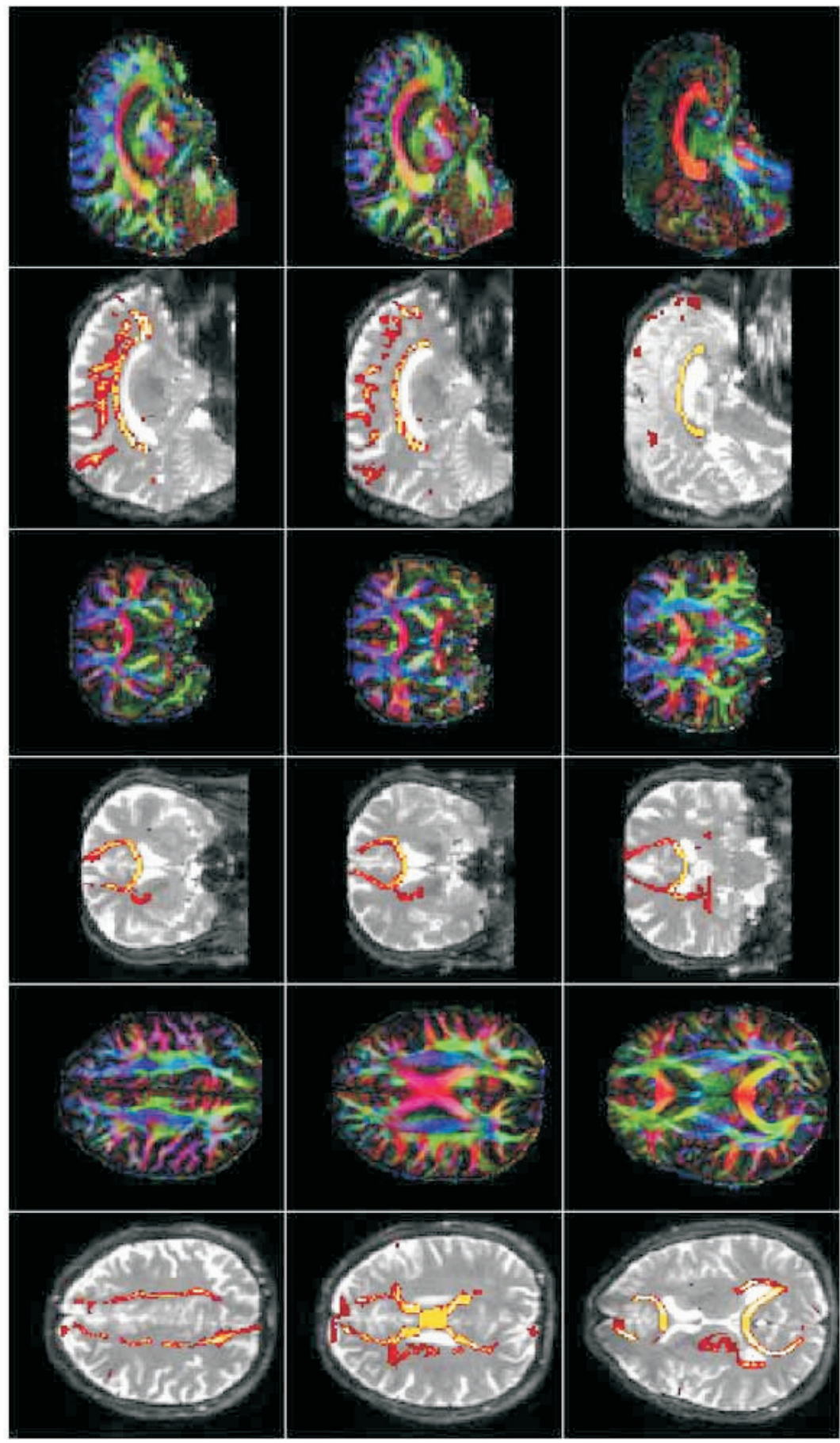

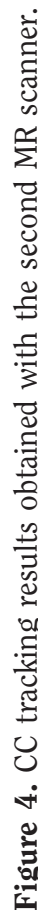




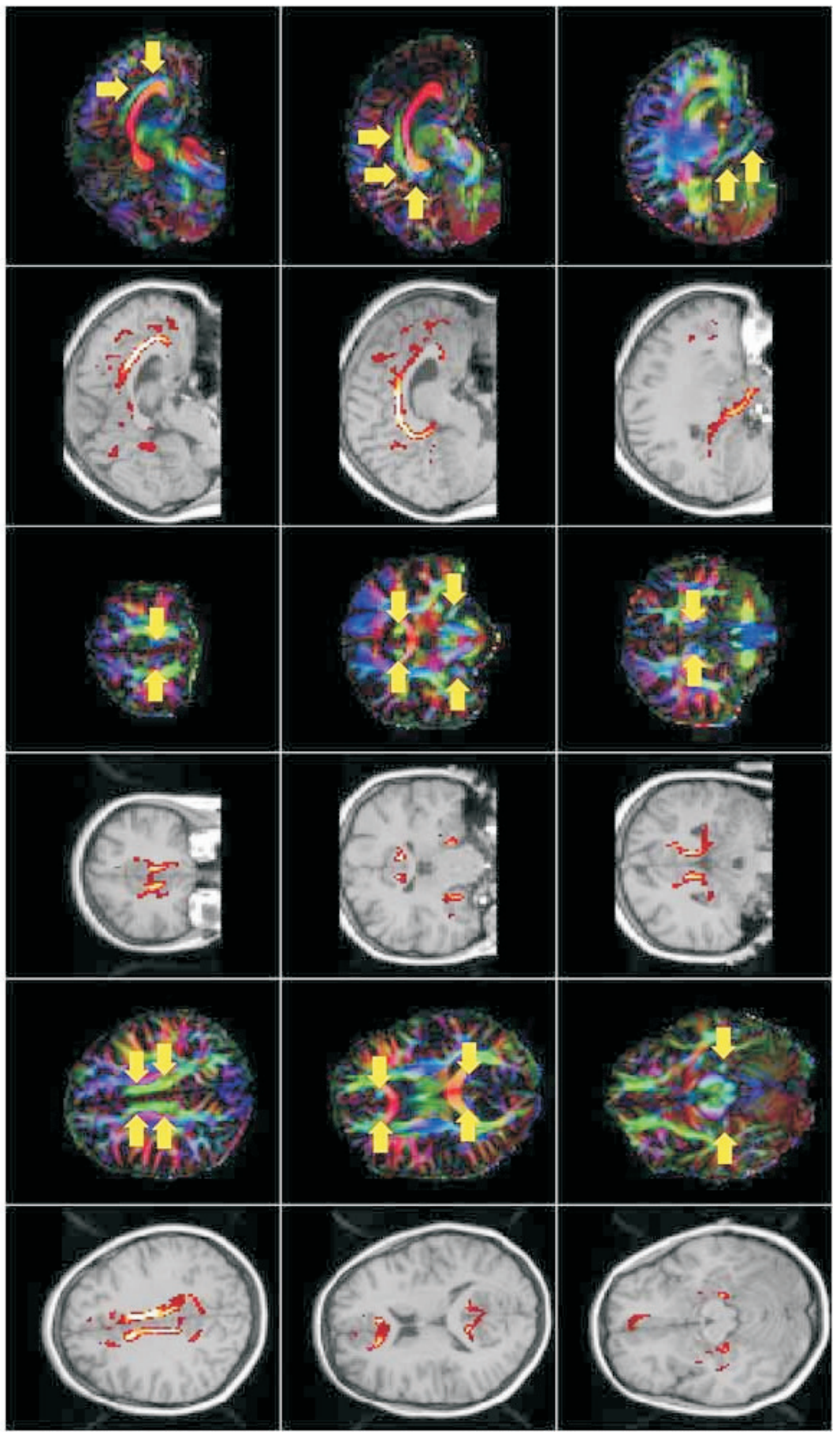

ఏ.] 


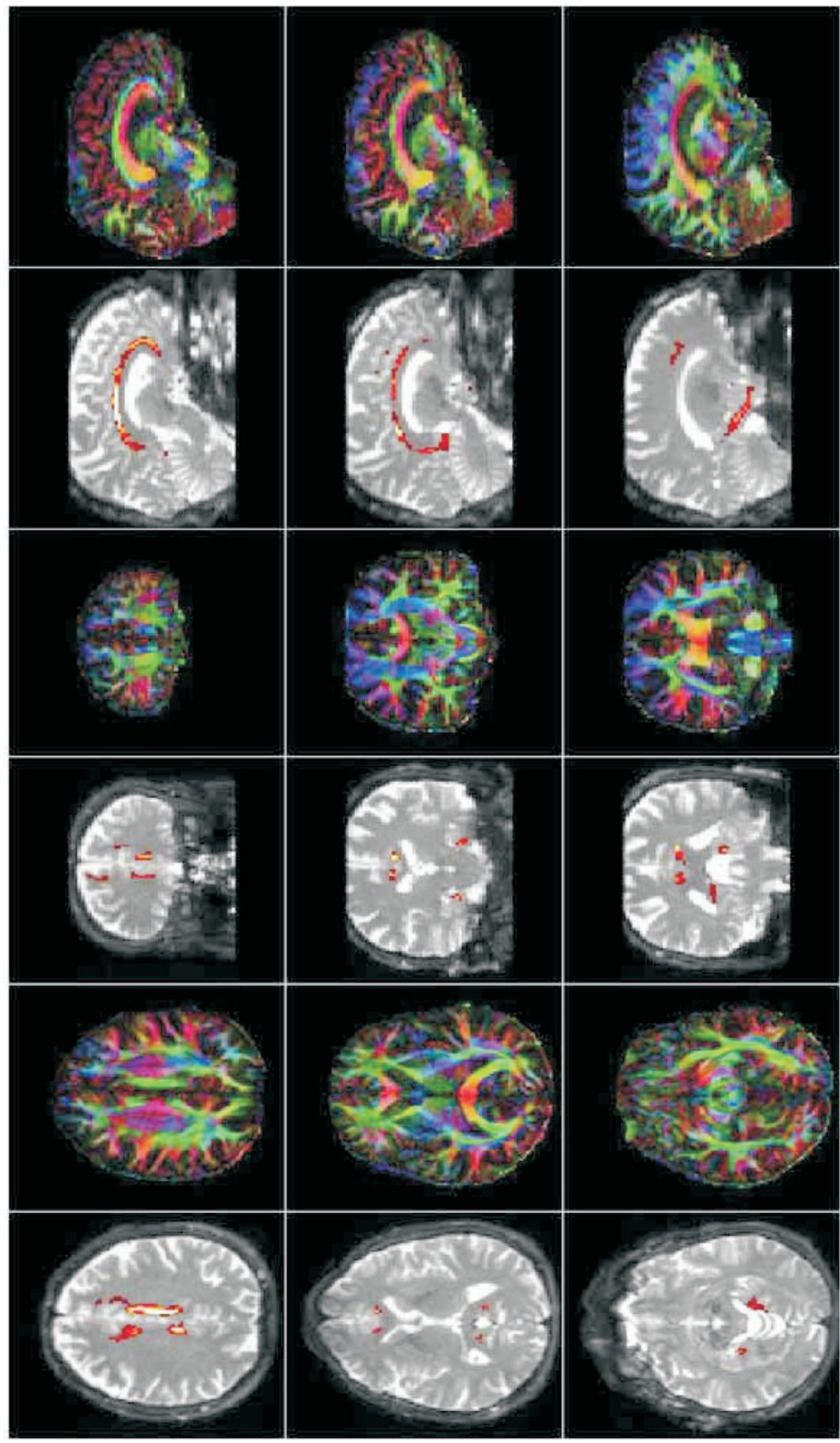

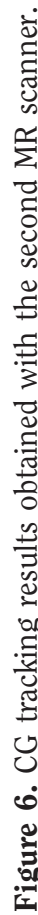


correctness. It is also interesting to notice the excellent correspondence between the obtained fibres and the colourmaps (yellow solid arrows).

In Figure 2 are depicted the results obtained for the same bundle but obtained with the second imaging unit. Since the representation of the results is the same, it is easy to compare them. Images show a good quality constancy if compared with them depicted in Figure 1 nevertheless the acquisition time is reduced by half.

In Figures 3 and 4 are depicted the $\mathrm{CC}$ reconstruction, while in Figures 5 and 6 are shown the results for CG fibres.

Considerations made for the CST are still valid for the $\mathrm{CC}$ and $\mathrm{CG}$ results. Reconstructed tracts show an high probability (they are composed by many bright voxels), a correct anatomical position and an excellent agreement with the colour-coded maps. Also in these cases the images quality is constant across the two imaging units.

\section{Conclusions}

Starting from the obtained results it is easy to notice that the chosen probabilistic reconstruction algorithm could represent an interesting alternative to the well-known deterministic methods (e.g. FACT, TEND [6, 4]. There still are some problems that have to be solved in order to exploit the described probabilistic method in clinical routine: the large amount of time needed to acquire the data and the long time period requested by the processing stage. The protocol optimization here developed provides a good starting point. In fact a total acquisition time of about 32 minutes is reasonable if DT acquisitions are the only aim of a diagnostic session. However, during the study, we tried to analyze the results obtained not only in the described ways, but also with 4 repetitions (32 minutes) for the first scanner and with 2 repetitions (16 minutes) for the second imaging unit. These alternative attempts gave us some hints that could lead to a further optimization in the data collecting time. The other obstacle lays in the large data processing time: this fact forces the medical equipe to perform images acquisition at least one day before the tractography stage. However if a CPUs array or a multicore system is available, the processing time can be reduced by a factor which is equal to the number of cores (e.g. with four $3.5 \mathrm{GHz}$ processors the time needed is about 2.5 hours). This work is preliminary for the applications in neurosurgery for the Epilepsy and Parkinson disease. Using Intera Achieva 1,5T we studied a patient during an Epilepsy surgery procedure to define the planning for deep electrodes implantation (SEEG 
procedure). However more detailed studies are necessary to verify the effective applicability in neurosurgery. The fact of making reliable to use a probabilistic algorithm in clinical practice translates in the possibility to make a quantitative comparison between different fibre tracking methods. About this point a preliminary attempt to validate probabilistic tractography with the deep electrodes information has been performed and it has lead to interesting results. It could be another good argument for future studies.

\section{Acknowledgements}

This work has been possible thank to the staff and equipments offered by Radiological Department of Niguarda Ca'Granda Hospital (Milano, Italy) and Philips Customer Visit Centre (Best, The Netherlands).

\section{References}

[1] Behrens TEJ, Johansen-Berg H, Jbabdi S, Rushworth MFS, Woolrich MW. Probabilistic diffusion tractography with multiple fibre orientations: What can we gain? NeuroImage. 2007; 34: 144-55.

[2] Behrens TEJ, Woolrich MW, Jenkinson M, Johansen-Berg H, Nunes RG, Clare S, Matthews PM, Brady JM, Smith SM. Characterization and Propagation of Uncertainty in Diffusion-Weighted MR Imaging. Magn Res Med. 2008; 50: 1077-1088.

[3] Jenkinson M, Smith SM. A global Optimization Method for Robuste Affine Registration of Brain Images. Medical Image Analysis. 2001; 5(2): 143-156.

[4] Lazar M, Weinstein DM, Tsuruda JS, Hasan KM, Arfanakis K, Meyerand ME, Badle B, Rowley HA, Haughton V, Field A, Alexander AL. White Matter Tractography Using Tensor Deflection. Human Brain Mapping. 2003; 18: 306-321.

[5] Minati L, Weglarz W. Physical foundations, models and methods of diffusion MRI of the brain: a review. Conc In Magn Reson Part A. 2007; 30A(5): 278-307.

[6] Mori S, Crain B, Chacko VP, Van Zijl PCM. Three Dimensional Tracking of Axonal Projections in the Brain by Magnetic Resonance Imaging. Ann Neurol. 1999; 45: 265-269.

[7] Smith SM, Jenkinson M, Woolrich MW, Beckmann CF, Behrens TEJ, Johansen-Berg H, Bannister PR, De Luca M, Drobnjak I, Flitney DE, Niazy R, Saunders J, Vickers J, Zhang Y, De Stefano N, Brady JM, Matthews PM. Advances in functional and structural MR image analysis and implementation as FSL. NeuroImage 2004; 23(S1): 208-219. (www.fmrib.ox.ac.uk/fsl/) 